\title{
Study of Filters through Programmable Instruments Implementing LabVIEW
}

\author{
Ms. P. Jyothi ${ }^{1}$, Mr. C. Chandra Mouli ${ }^{1}$, Prof. C. Nagaraja ${ }^{2}$, \\ Prof. K. Nagabhushan Raju ${ }^{2}$ \\ ${ }^{I}$ Research Scholar of InstrumentationDepartment, Sri Krishnadevaraya University, Anantapur, A.P., INDIA \\ ${ }^{2}$ Department of Instrumentation, Sri Krishnadevaraya University, Anantapur, A.P., INDIA
}

\begin{abstract}
The present work describes the detailed study of the active filters and its characteristics by interfacing the programmable instruments with personal computer $(P C)$ using LabVIEW. The programmable instruments used in the present work are function generator from Protek and HP digital multimeter. Two serial ports are used to establish the communication between function generator, digital multimeter and PCthrough the virtual instrument (VI) developed using LabVIEW software tool. Programmable function generator produces the continuous frequencies to one of the filter circuit using LabVIEW program through one serial communication port and the corresponding output voltage of the filters along with the input frequency is stored in a file by reading the output $A C$ voltage from the digital multimeter through another serial communication port using LabVIEW programming. The workprovides the efficient study of first and second order Active filters with high precession.
\end{abstract}

Keywords: Digital Multimeter, LabVIEW, PC, Programmable Function Generator, Virtual Instrument.

\section{INTRODUCTION}

An electric filter is a frequency selective circuit that either allows a specified band of frequencies or attenuates particular signal of frequencies [1]. Filter circuit can be designed using passive elements (resistor, capacitor, inductor) called Passive filter and also the combination of active and passive elements known as Active filters. Active filters are more economical than passive filters, easy to tune, does not cause loading of the source. Filters are most extensively used in the field of communications, signal processing and commonly used in sophisticated electronic systems like radio, TV, radar, space satellites and biomedical instrumentation [1].

In practice it creates some difficulty to study the filter characteristics by continuously varying of frequency manually and measuring the corresponding voltage. To overcome this problem the present work was aimed to design an integrated instrumentation system which consists of programmable instruments for the practical purpose that are used in labs.

The programmable instruments are meant to use in the PC based instrumentation. The former instrumentation techniques are used to interface the programmable instruments with the PC through the programmable languages which doesn't provide Graphical User Interface (GUI) to observe and to feed the input values. The programmable languages C, BASIC, etc. are used to provide the Standard Commands for the Programmable Instruments (SCPI). SCPI written in programming languages are very difficult to analyze and to modify the code according to the application requirement.

In the present work the language used to program the instruments is graphical programming language which provides a GUI to give the input values for programmable instruments. A programmable instrument with concurrent programming, Graphical user interface, and real time systems integrates in the current work [7]. The programmable instrument i.e. digital synthesis arbitrary function generator/counter (Protek 9305) and HP digital multimeter (Hewlett Packard 34401A) were used in the present system. The applications of these instruments are most extensively functional in industries and research. By implementing set of graphical programming sub routines under LabVIEW environment, one can enhance the full-fledged system in order to monitor the flow of data continuously through the interface bus using developed integrated software. Programmable function generator and HP digital multimeter were connected to two comports to develop serial communication for writing frequency to the function generator and reading $\mathrm{AC}$ voltage from the Digital multimeter using LabVIEW programming. In this system, programmable function generator and a digital multimeter were connected in a closed loop through personal computer. 


\subsection{Hardware Design}

\section{INSTRUMENTATION}

The hardware design of the present work describes the interfacing of the function generator, digital multimeterand filter circuits with PC. PC used in the present work is DELL OptiPlex 380, which comes with only one serial port and the second serial port is established with one of the USB ports. The default serial port was used to interface the digital multimeter and the serial port through the USB port was used to interface the function generator. A USB to RS232 converter was used to convert the USB port to serial port along with the driver.

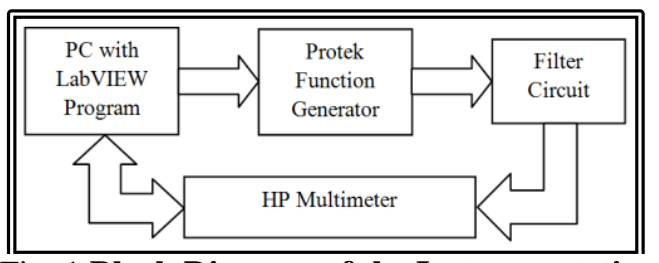

Fig. 1.Block Diagram of the Instrumentation System

Figure 1 shows the block diagram of the instrumentation system. The block diagram shows that the interface was done in closed loop fashion. The USB serial port was used to interface the protek function generator which uses only three lines from the serial port i.e.pin2 and pin3 of DB9 connecter at PC end were connected to the pin 3 and pin 2 of the function generator end respectively for transmitting the commands. Pin5 of both PC and instrument was shorted as it acts as common ground and the rest of the pins are NCs [2].

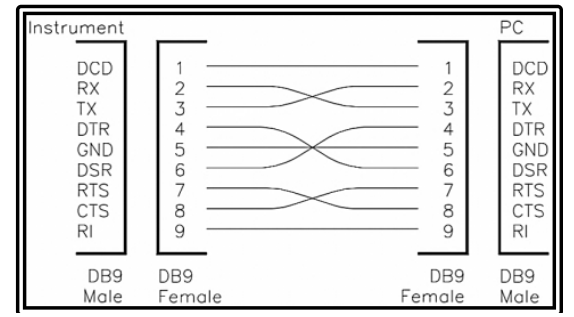

Fig. 2.Pin Configuration of PC and Digital Multimeter

Figure 2 shows the pin configuration of interfacing of the digital multimeter with PC. Pins 2, 3, and 5 was connected same as the function generator. Pins 1 and 9 were connected to the same pins in the both ends. Pins 4, 5, 7 and 8 at instrument end were connected to the pins 5, 4, 8 and 7 at PC end respectively [3]. For both instruments, front panel was used to connect it with the filter circuits. In the present study function generator is used as an input instrument that is programmed to give continuous incremental frequency with constant peak to peak voltage. The digital multimeter is the output instrument that is programmed and configured to read the AC voltage.

The present work studies the characteristics of low pass,low pass ( $1^{\text {st }}$ order $)$, high pass, high pass $\left(1^{\text {st }}\right.$ order), band pass and band reject filters. Figure 3 shows the schematic circuit diagram of active low pass filter.The low pass active filter is designed by connecting an invertingamplifier to the basic RC low pass filter.The inverting amplifier is connected with unity gain. The operational amplifier OP07 was used which works with +12 and $-12 \mathrm{~V}$. All the resistors values are $1 \mathrm{~K}$ and capacitor value is $0.01 \mathrm{uF}$. The $\mathrm{AC}$ signal from the function generator is connected to the input pins 1 and 2 of the circuit [3].

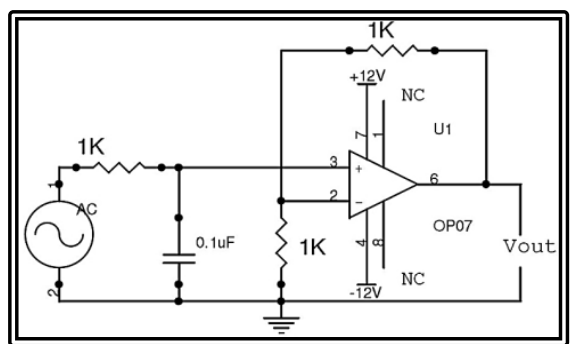

Fig. 3.Schematic Circuit Diagram of Active Low Pass Filter

PC is connected to the function generator through USB serial port to feed the continuous frequency signal to the filter circuit and the low pass filter circuit allows the low frequencies and rejects the high 
frequencies. The output AC voltage is measured using digital multimeter and stored in a file. The next section discusses about the software design of the present study.

\subsection{Software Design}

The software design describes the implementation of LabVIEW to study the filter characteristics. LabVIEW is asoftware tool that uses graphical programming language for data acquisition and instrument control. The flexibility to analyze instrumentation made it to use in the present study [5]. LabVIEW includes conventional program development tools, so that user can set breakpoints, animate program execution to see how data passes and single-step through the program to make debugging and program development easier. LabVIEW contains application-specific libraries for general purpose interface bus (GPIB), serial instrument control, data analysis, data presentation and data storage [5]. Depending up on the application, external interfaces are integrated with LabVIEW. External interfaces like function generator, digital oscilloscope and digital multimeter are used for data acquisition through serial communication port.

In the present work complete software is developed in LabVIEW for function generator and digital multimeter. Figure 4 and 5 shows the front panel and block diagramof the LabVIEW VI of the present work respectively.

Front panel is used as GUI and to configure the settings for serial port operation. VISA Resource Nameand VISA Resource Name2are VIs which were used to select the com port in the available comport list. Baud rate, data bits, parity, stop bits and flow control are 32 bit unsigned long integer data types which are used to configure the serial port. The input frequency and number of iteration are 32 bit integer data types which are used to give the input frequency, and the count increment value and number of iterations for thefor loop.The cluster Serial Configuration is used to set the values that are required for serial communication like data bits, baud rate and parity. Function (0: DC Voltage) is 32 bit integer data type which is used to select the output type of the digital multimeter. Write data is a string indicator which shows the input frequency that is feeding to thefunction generator. Measurement is a 64 bit double data type which is used to show the measured AC voltage onthe front panel.

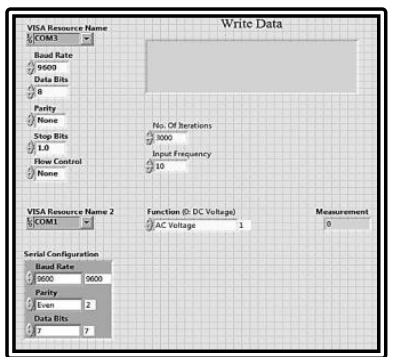

Fig. 4.Front Panel of LabVIEW VI

The block diagram adds the code in graphical representation of functions to control the front panel objects. The block diagram contains the graphical source code, also known as $\mathrm{G}$ code or block diagram code. Front panel objects appear as terminals on the block diagram. Figure 5 shows the block diagram which the G code for the present work. The functions used in the present study to write the data to the function generator are VISA Configure Serial Port VI,VISA Write Function,Property Node, VISA Close Function, Number to Decimal String Function and Concatenate String Function. VISA Configure Serial Port VI initializes the serial port specified by VISA resource name to the specified baud rate, data bits, parity, stop bits and flow control settings. VISA Write Function writes the string of commands from Input Frequency and from two constants from the interface specified by VISA resource name.

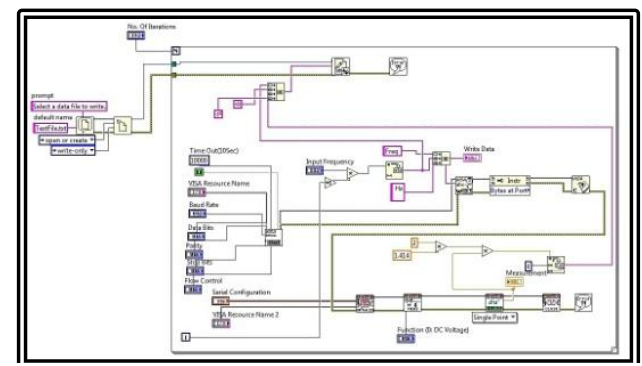

Fig. 5.Block Diagram of LabVIEW VI

The Property Node adapts to the class of the objects that wasreferred by the user. Property nodes are preconfigured in LabVIEW library to access XML Properties, VISA properties, .NET properties, and ActiveX 
properties.VISA Close Function closes the event object specified by the VISA Resource Name. Number to Decimal String Function converts the numbers to the decimal string. Concatenate String Function appends the group of strings to one collective string.

Digital multimeter is configured by using the Agilent 34401 device drivers provided by the National Instruments. The Agilent's device drivers Initialize.vi, Measure.vi, Read(Single Point).vi and Close.vi are used in the present work.Initialize.vi establishes the connection between the PC and instrument with the specified settings. Measure.vi configures the measurement type, resolution and range on the instrument. Read (Single Point).vireads and return a single measurement from the instrument.Close.vi performs an instrument error query and terminates the software connection to the instrument.

The function generator write and digital multimeter read were kept in for loop in the block diagram of the LabVIEW program and the contents of input frequency and output AC voltage wasgoing to be saved to the file.

\section{WORKING}

Programmable instruments are interfaced with personal computer using SCPI (Standard Commands for Programmable Instruments) through RS232 standard interface through one of the filters like active low pass, active high pass, Band pass or Band reject circuit. The functionality of instruments is controlled using LabVIEW programming in a personal computer. The function generator is configured with the baud rate 9600 , 8-data bits, 1 STOP bit andnone parity. In the same way Digital multimeter is also configured with 7 data bits, 9600 baud rate and even parity.

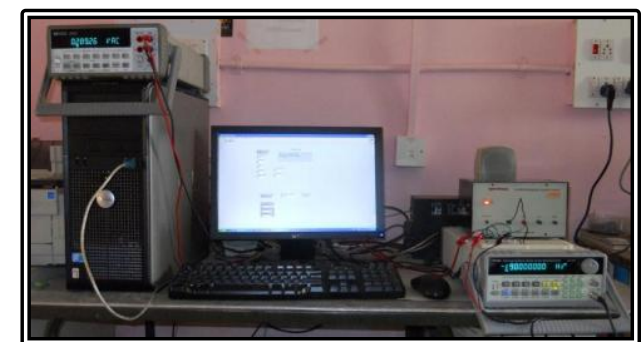

Fig. 6.Photograph of the Instrumentation System

At the power-on software initializes the function generator and digital multimeter, which is connected to the serial com ports COM3 and COM1 respectively. The input frequency and number of iterations is given as inputs from the front panel of the LabVIEW VI.The input voltage is given manually. The frequency with $1 \mathrm{~V}$ peak-to-peak voltage is given as input to the active low pass filter circuit. In the present study the input frequency is given as $10 \mathrm{~Hz}$ and the number of iterations as 1200 . The iteration count is multiplied with the input frequency for each iteration of the for loop. Therefore the frequency starting from $10 \mathrm{~Hz}$ to $12 \mathrm{KHz}$ is passing through the filter circuit with a frequency difference of $10 \mathrm{~Hz}$ for each iteration.The digital multimeter is configured in such a way that the multimeter is going to read the AC voltage. The total frequencies and the corresponding $\mathrm{AC}$ voltages are stored to the text file. The stored values are copied and then draw the graph in the excel sheet.

\section{RESULTS AND CONCLUSION}

The characteristics of the filters are studied after making the appropriate filter circuits and LabVIEW software. The study of characteristics of active filters with the integrated instruments was tested. The low pass $\left(1^{\text {st }}\right.$ order $)$, high pass $\left(1^{\text {st }}\right.$ order $)$ circuits and its response curve were shown in figure 7 . The band pass and band reject circuits and its response curves were shown in figure 8 . The low pass $\left(2^{\text {nd }}\right.$ order $)$ and high pass $\left(2^{\text {nd }}\right.$ order $)$ filters circuits and response curves are shown in figure 9. The results obtained are compared with the manual varying frequency measurementand the results shows are satisfactory with the existing method.

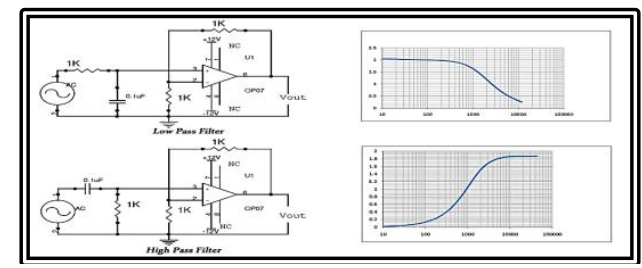

Fig. 7.Low Pass Filter, High Pass Filter and Response Curves 


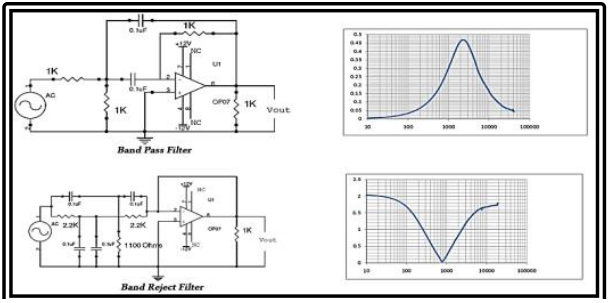

Fig. 8.Band Pass Filter, Band Reject Filter and Response Curves

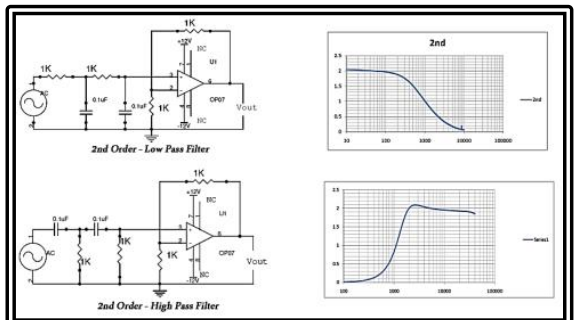

Fig. 9.2 ${ }^{\text {nd }}$ Order Low Pass Filter, $2^{\text {nd }}$ Order High Pass Filter and Response Curves

\section{REFERENCES}

[1] H S Kalsi, Electronic Instrumentation (Tata McGraw-Hill Publishing Company Limited, 2005).

[2] Digital Synthesized Function Generator/Counter (Protek 9300 series) User Manual.

[3] Digital Multimeter (Hewlett Packard 34401A) User Manual.

[4] S.V.Subrahmanyam, Linear And Digital IC's Applications (HI-TECH Publishers)

[5] Jeffrey Travis \& Jim Kring, LabVIEW For Everyone (Pearson Education, Prentice Hall, 2007)

[6] Cory L. Clark, LabVIEW Digital Signal Processing (Tata McGraw-Hill Publishing Company Limited, 2005).

[7] M. Ashok Kumar, B. Rama Murthy, Ch.V.V. Ramana, Willem Clarke and K. VenuMadhav, Capacitance Measurement with Integrated Instruments, 2011 International Conference on Recent Advancements in Electrical, Electronics and Control Engineering, 978-1-4577-2149-6/11/\$26.00 @ 2011 IEEE.

[8] C. Chandra Mouli, P. Jyothi, K. Nagabhushan Raju, C. Nagaraja, T. Bheema Lingaiah and M. Ashok Kumar,Implementation of serial communication in LabVIEW to interface with 8051 based microcontroller, Research Journal of Engineering \& Technology, Vol.3. Issue.4, pp.7-9, ISSN 0974-2824.

[9] Muhammad Ali Mazidi, Janice Gillespie Mazidi and Rolin D. McKinlay, The 8051 Microcontroller and Embedded Systems using Assembly and $C,\left(2^{\text {nd }}\right.$ EditionPHI $)$

[10] C. Nagaraja, C. Chandra Mouli, S. Athavulla, and T. Bheemalingaiah, "A Microcontroller Based Programmable Power Supply." Lab Experiments - A Journal of Laboratory Experiments, vol. 10, No - 4, pp.249-253, December 2010.

[11] C. Chandra Mouli, V. Ramnath, D. Sailaja, and K. Nagabhushan Raju, "Embedded System Based ExhaustFan Control.'LabExperiments - A Journal of Laboratory Experiments, vol. 11, No - 3, pp.200-201, September 2011. 